International Journal of Neural Systems, Vol. 28, No. 9 (2018) 1850019 (13) pages)

(C) The Author(s)

DOI: 10.1142/S0129065718500193

\title{
Two-Source Validation of Progressive FastICA Peel-Off for Automatic Surface EMG Decomposition in Human First Dorsal Interosseous Muscle
}

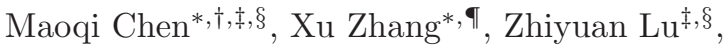 \\ Xiaoyan $\mathrm{Li}^{\ddagger, \S}$ and Ping Zhou ${ }^{\dagger, \ddagger, \S, \|}$ \\ *Biomedical Engineering Program \\ University of Science and Technology of China \\ Hefei, P. R. China \\ ${ }^{\dagger}$ Guangdong Work Injury Rehabilitation Center \\ Guangzhou, P. R. China \\ ${ }^{\ddagger}$ Department of Physical Medicine and Rehabilitation \\ University of Texas Health Science Center, Houston, USA \\ $\S T I R R$ Memorial Hermann Hospital, Houston, USA \\ 『Xuzhang90@ustc.edu.cn \\ "IPing.zhou.1@uth.tmc.edu
}

Accepted 20 April 2018

Published Online 14 June 2018

\begin{abstract}
This study aims to assess the accuracy of a novel high density surface electromyogram (SEMG) decomposition method, namely automatic progressive FastICA peel-off (APFP), for automatic decomposition of experimental electrode array SEMG signals. A two-source method was performed by simultaneous concentric needle EMG and electrode array SEMG recordings from the human first dorsal interosseous (FDI) muscle, using a protocol commonly applied in clinical EMG examination. The electrode array SEMG was automatically decomposed by the APFP while the motor unit action potential (MUAP) trains were also independently identified from the concentric needle EMG. The degree of agreement of the common motor unit (MU) discharge timings decomposed from the two different categories of EMG signals was assessed. A total of 861 and 217 MUs were identified from the 114 trials of simultaneous high density SEMG and concentric needle EMG recordings, respectively. Among them 168 common (MUs) were found with a high average matching rate of $(96.81 \pm 3.65) \%$ for the discharge timings. The outcomes of this study show that the APFP can reliably decompose at least a subset of MUs in the high density SEMG signals recorded from the human FDI muscle during low contraction levels using a protocol analog to clinical EMG examination.
\end{abstract}

Keywords: Surface EMG; automatic decomposition; progressive FastICA peel-off; two-source validation.

\section{Introduction}

By separating electromyogram (EMG) signal into its constituent motor unit action potential (MUAP) trains, EMG decomposition provides MUAP waveform and motor unit (MU) discharge information, thus playing a fundamental role for the investigation of motor control and examination of neuromuscular diseases 14 The results of EMG decomposition can also be used to estimate the user's intention, thus having a potential for the development of a novel human-machine interface ${ }^{5}$ to enrich or supplement the already existing ones $[6]$ Most of the previous

This is an Open Access article published by World Scientific Publishing Company. It is distributed under the terms of the Creative Commons Attribution 4.0 (CC-BY) License. Further distribution of this work is permitted, provided the original work is properly cited. 
EMG decomposition methods target intramuscular (needle or fine wire) EMG at relatively low muscle contraction levels, where template matching is usually used to discriminate MUAPs from different MUs. Considerable efforts have also been focused on the separation of superposed MUAP waveforms to obtain the MU firing behaviors 1013

Compared with intramuscular EMG (IEMG), decomposition of surface EMG (SEMG) is much more difficult because of the excessive MUAP superposition (even at low muscle contraction levels, due to a large number of recorded MUs and longer MUAP duration), and less distinguishable MUAP waveforms from different MUs (due to low-pass filtering effects of skin and subcutaneous body tissues)! 14 While decomposition of single channel regular SEMG is usually not a feasible task, recent development in high density SEMG significantly promotes SEMG decomposition. On one hand, high density SEMG allows simultaneous recording of up to hundreds of channels of SEMG using electrode arrays comprised of a number of tiny electrode probes with small interprobe distance. $\frac{15}{18}$ The spatial information provided by an electrode array can effectively overcome the MUAP similarity in single channel recording, given the unique location of each MU within a muscle. Thus, two-dimensional MUAP template matching can be applied for SEMG decomposition at low muscle contraction levels $\frac{19}{}$ On the other hand, to solve excessive MUAP superposition and enable high density SEMG decomposition at higher muscle contraction levels, more advanced blind source separation (BSS) techniques can be applied, among which representative advances include convolution kernel compensation $(\mathrm{CKC})^{20} \frac{22}{22}$ and progressive FastICA peel-off (PFP) $\underline{\underline{23.24}}$

The PFP and its automatic implementation, i.e. automatic PFP (APFP), were recently developed by our laboratory ${ }^{23124}$ The proposed framework uses a popular BSS technique called independent component analysis (ICA), 25 which has been widely applied to process different biological signals. $\frac{26}{30}$ As one of the most popular and effective ICA algorithms, FastICA $^{31}$ has been used for SEMG decomposition in the previous studies, but with limited success ${ }^{32}[35$ However, by combining FastICA with other advanced signal processing techniques, our group has developed the PFP framework and its automatic implementation (i.e. APFP), which have been proved to be able to significantly promote the decomposition performance. $23|24| 36$

When a method is proposed to decompose an EMG signal, it is incumbent to prove that the decomposition is performed accurately. A simulation approach based on mathematical modeling of the EMG signal is most often used for the assessment of the decomposition performance. By adjusting a range of input components (such as MU number, firing rate, size, location, noise level, etc.) to the EMG model, the decomposition performance under different conditions can be quantitatively analyzed. However, although the simulated EMG signals can be very close to experimental ones with advances in (both intramuscular and surface) EMG simulation, $37 \sqrt[40]{4}$ simulated EMG signals are not equal and cannot ultimately replace the experimental EMG for the evaluation of the decomposition performance. Therefore, it still remains a limitation for the simulation-based assessment that the reliability of the decomposition accuracy depends on the realism of the simulation.

For a real or experimental EMG signal, the composition is not known a priori. An often used evaluation approach for IEMG decomposition, typically at low muscle contraction levels, is to compare the decomposition result of the computer-based methods with the visual judgement of an experienced human operator. The expert's judgment can usually be trusted when there is no or little MUAP superposition contained in the IEMG signal. However, with increased MUAP superposition levels it becomes more and more difficult to rely on visual judgement. Such a visual assessment approach is even more challenging or impractical for SEMG decomposition due to heavy MUAP superposition and similar MUAP waveforms recorded from the skin surface.

To validate the SEMG decomposition performance with experimental signals, a more objective method is the so-called "two-source" validation, which requires simultaneous recording of SEMG and IEMG signals. $\frac{41}{1}$ Then, the IEMG signal can be decomposed using already well-established methods, and the SEMG can be independently decomposed using the proposed methods. It is expected that MUs contributing to both signals (i.e. common MUs) can be recorded. Cross-checking of the agreement on the timing of the common MUs discharges 
can give an estimation of the decomposition accuracy. If the independent decomposition of two separate signals agrees on the timing of a particular MU discharge, we think they are both correct. Otherwise, they would both have to involve an error of exactly the same amount. The probability of this situation arising is very small. The two-source method has been widely accepted for assessment of the decomposition performance when a novel SEMG decomposition method was proposed. For example, the accuracy of using the CKC approach for highdensity SEMG decomposition has been validated by the two-source method. ${ }^{42 / 43}$ Two-source validation has also been performed by $\mathrm{Hu}$ et al. for the dEMG (previously referred to as PDsEMG) algorithm based on SEMG recordings from a five-pin sensor array 44

For the APFP method, we have performed a series of validation for its decomposition performance, starting from high density SEMG simulations. ${ }^{23 \mid 24}$ We have also compared its decomposition performance with the CKC approach $\sqrt[36]{ }$ In this study, we further quantitatively assess the performance of the APFP for high-density SEMG decomposition using the two-source method $\stackrel{41]}{\text { The findings }}$ from the two-source validation demonstrate that the APFP can reliably decompose at least a subset of MUs in high-density SEMG signals recorded from the first dorsal interosseous (FDI) muscle during low contraction levels, using a protocol analog to that often used in clinical EMG examination.

\section{Methods}

\subsection{Experiments and protocols}

\subsubsection{Participants}

Five neurologically intact subjects (mean $\pm \mathrm{SD}$, age: $36 \pm 8$ years, all male) who did not have any history of neural or muscular disorders were recruited to participate in this study. A chronic stroke subject (age: 65 years, five years post-stroke, female) also participated in the study. All subjects gave written informed consent before any experiment procedures. The experimental protocols were in accordance with the Declaration of Helsinki, and approved by the Committee for the Protection of Human Subjects (CPHS) of the University of Texas Health Science Center at Houston and the TIRR Memorial Hermann Hospital (Houston, TX, USA).

\subsubsection{SEMG and IEMG recordings}

During the experiment, each subject was comfortably seated with the investigated arm pronated and placed on a height-adjustable table. High-density SEMG and IEMG were recorded from the FDI muscles. Among the five neurologically intact participants, the recordings were performed bilaterally for four subjects, and in the dominant hand for one subject. For the stroke subject, the recordings were performed in the paretic FDI muscle of the affected side. Therefore, a total of 10 FDI muscles were recorded. Prior to electrode placement, the skin of the examined regions was prepared with gentle abrasion and cleaned using alcohol pads.

SEMG was recorded by a flexible two-dimensional 64 -channel $(8 \times 8$, individual recording probe $1.2 \mathrm{~mm}$ in diameter, center-to-center distance $4 \mathrm{~mm}$ ) electrode array (TMS International BV, The Netherlands). The reference electrode was located near the elbow. Before experiment, the flexible surface electrode arrays were penetrated with several holes in different locations to enable the insertion of a concentric needle. This was performed very carefully not to break the wiring of the flexible electrode array. After gel preparation, the flexible surface electrode array was attached to the FDI muscle with a compatible double-sided adhesive sticker. The electrode array was further secured with a medical tape (while leaving the holes uncovered). A Refa 128-channel amplification system (TMS International BV, The Netherlands) was used to record the electrode array SEMG signals. The signals were sampled at $2 \mathrm{kHz}$ per channel, with a band pass filter setting at $10 \mathrm{~Hz}^{-}$ $500 \mathrm{~Hz}$.

IEMG recordings were carried out using a Natus UltraPro S100 EMG system (Natus Neurology Inc., Middleton, WI, USA). The recordings were performed using a conventional concentric needle electrode with a diameter of $0.58 \mathrm{~mm}$ and a recording area of $0.07 \mathrm{~mm}^{2}$ (Alpine bioMed, Denmark). A surface reference electrode was placed on the back of the hand near the ulna styloid. After placing the flexible high density surface electrode array on the tested muscle, the concentric needle electrode was carefully inserted into the muscle through one of the penetrated holes of the flexible array. The concentric needle EMG was sampled at $44,100 \mathrm{~Hz}$ with a band pass filter setting at $10 \mathrm{~Hz}-10 \mathrm{kHz}$. 
Both high-density SEMG and concentric needle EMG recordings were displayed in real-time on computer monitors and stored in hard drives for decomposition analyses.

\subsubsection{Protocols}

In this study, simultaneous 64 channels of highdensity SEMG and one channel of concentric needle EMG signals were recorded using a strategy commonly used in clinical EMG examination. We did not apply a standard laboratory load cell setup to monitor force and require subjects to perform precise muscle force control at different contraction levels, as described in our previous studies ${ }^{45,46}$ Instead, we provided a resistance force to the examined muscle and instructed the subject to first perform an isometric contraction at a low-force level. During the recording, the concentric needle electrode was positioned stable by the examiner (Fig. 1). Once the clear-cut isolated single MU activities which appeared on the screen of the concentric needle EMG were visually identified, the subject was encouraged to try to maintain the contraction level for at least $10 \mathrm{~s}$, preferably up to $1 \mathrm{~min}$, during which both high density SEMG and concentric needle EMG were recorded. During the experiment, the subject had visual feedback by watching the EMG signals displayed on the computer monitor. Verbal encouragement was also provided to the subject to help maintain the contraction.

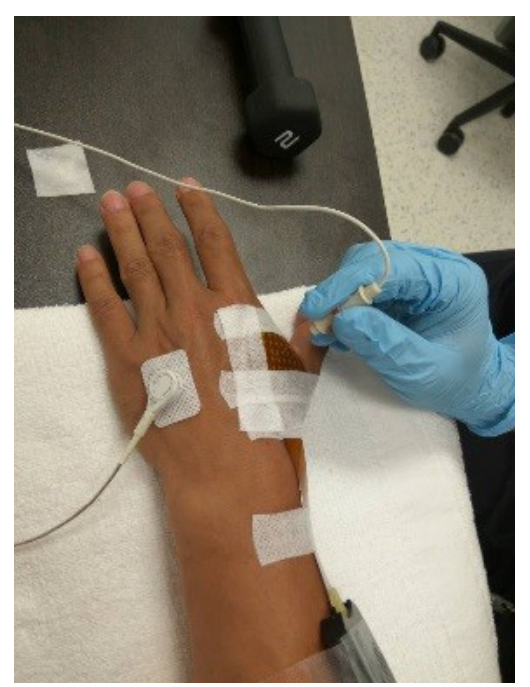

Fig. 1. Simultaneous recording of high density SEMG and concentric needle EMG signals from the FDI muscle.
For each subject, multiple trials were recorded by advancing the concentric needle to different depths or positions for each of the insertion sites. To avoid causing discomfort to subjects, no more than three needle insertions (one insertion at a time, through different penetrated holes of the flexible surface electrode array) were allowed for each of the tested muscles. Different FDI muscle contraction patterns (e.g. index finger abduction and/or flexion) were performed, so a sufficient number of MUs can be identified. Substantial rest time between trials was provided to prevent potential mental or muscle fatigue during the experiment.

For each trial, an electrical stimulation protocol was used to help synchronize the high-density SEMG and the concentric needle EMG signals for the data processing. The subject was instructed to relax the muscle after completing a trial of contraction. The ulnar nerve was stimulated about $2 \mathrm{~cm}$ proximal to the wrist crease. The duration of the single pulse stimulation was $200 \mu \mathrm{s}$. The stimulation intensity varied across different subjects (which was sufficient to elicit a visible $M$ wave response for most trials). The recorded stimulus artifacts can serve as an alignment marker for synchronization of the high density SEMG and the concentric needle EMG signals.

\subsection{Data analysis}

\subsubsection{SEMG decomposition}

The high density SEMG signals were automatically decomposed by the APFP framework, which performs automatic implementation of the PFP ${ }^{24}$ The APFP framework can be viewed as a process of progressively expanding the set of MU spike trains. In the framework, the initial set of MU spike trains can be estimated by applying FastICA ${ }^{31}$ to high density SEMG. Then, a peel-off procedure is employed to subtract the estimated MUAPs of the identified MUs from the original signal. Such a procedure mitigates the effect of the already identified MUs on the FastICA convergence, so more MUs can emerge when processing the residual signal again with FastICA. In order to ensure the reliability of the extracted MU spike trains, a series of automatic screening and revision steps are performed before the peel-off procedure. For each output of FastICA, a valley-seeking clustering method ${ }^{47}$ is used to extract and cluster the spikes. Then a constrained FastICA is applied 
to assess each clustered spike train and correct possible erroneous or missed spikes. And multiple constrain parameters are proposed for the final screening of the spike trains according to the MU firing behavior. The framework is iterative by repeatedly running the peel-off procedures until no additional new MUs can emerge. Briefly, the implementation of the APFP framework is outlined in Appendix A. For a more detailed description of the APFP framework (including parameter settings), the reader can refer to Ref. 24

\subsubsection{IEMG decomposition}

The concentric needle EMG signals were independently decomposed using a computer-based method, with operator manual intervention. The decomposition method is a combination of the peel-off strategy and the valley-seeking clustering 47 (Appendix B), as described below.

(1) If there exists a MU with a distinctive amplitude (compared to other MUs), set an appropriate threshold to extract this MU; then peel it off from the original signal and update the residue. Repeat this step until one cannot find such kind of MUs.

(2) If there exist several MUs with distinctive amplitudes (compared to the baseline), set an appropriate threshold to extract the spikes, then use the valley-seeking clustering method to sort out the spikes. Those clusters that demonstrate a clear clustering center can be judged to belong to one MU. If the cluster fails, end the procedure. Otherwise, peel them off from the original signal and update the residue, then go back to step (1).

This strategy is straightforward to understand. The MUs are identified based on their magnitudes in the descending order. In this process, we always first extracted the MUs with distinctive amplitude which are most reliable (i.e. they are hardly affected by any superposition). After peeling these easilyidentified MUs, we then used the valley-seeking clustering method to seek the remained MUs. The root mean square (RMS) and the difference absolute standard deviation value (DASDV) were used as clustering features $\stackrel{48}{4 a l l e y-s e e k i n g ~ c l u s t e r i n g ~ c a n ~ a c h i e v e ~}$ a good result if there are only a few superpositions.
However, if these MUs contain too many superpositions, the clustering result is not meaningful. This can be judged by whether clear cluster centers can be found. The clusters with dispersed feature distributions should be discarded.

Note that in our decomposition of the IEMG, the only processing that can deal with the superposition is the peel-off procedure. MUs with superposition may emerge after the peel-off steps. Otherwise, the superposed MUs have to be excluded from further processing. In order to ensure the two-source validation accuracy, the concentric needle EMG analysis was limited to extraction of the sufficiently reliable MUs, so by comparing with these MUs we were able to obtain a firm estimation of the accuracy of the high-density SEMG decomposition.

\subsubsection{Calculation of matching rate}

Although we used the electrical stimulation protocol to generate an alignment marker, we had to further align the signals because the difference in sampling rate and the width of the electrical stimulus imposed a difficulty in finding the precise trigger time. After a rough alignment using the electrical stimulation marker, we applied a down sampling and shift inner product matching strategy to compare the two spike trains in different sampling rates. To measure the matching degree of two spike trains, the matching rate $(\mathrm{MR})$ was calculated 36 .

$$
\mathrm{MR}=\frac{2 \cdot N_{\mathrm{COM}}}{N_{I}+N_{S}} \cdot 100 \%,
$$

where $N_{I}$ and $N_{S}$ are the total number of the spikes from the concentric needle EMG spike train and the SEMG spike train, respectively. $N_{\mathrm{COM}}$ stands for the number of the common spikes. In this study, we accepted two spikes from different spike trains as corresponding spikes when they were located within $\pm 1 \mathrm{~ms}$.

In this process, two systems can be precisely aligned during calculation of the MRs (if they have common units). In order to reduce the computational load of shift inner product, we further considered that practically the errors caused by stimulus alignment would not exceed $1 \mathrm{~s}$. Therefore, when calculating the MR, it was sufficient to perform the alignment of the two systems within a $1 \mathrm{~s}$ neighboring period of a marker. For each spike train of the 
concentric needle EMG, we used the following procedures to find out its corresponding spike train of the electrode array SEMG and calculated their MRs:

(1) Convolute the spike train of the concentric needle EMG with a $2 \mathrm{~ms}$ unit rectangular window, which extends it to a square wave train.

(2) Down sample the square wave train to the same sampling rate of the SEMG signal, named $T_{I}$.

(3) Take $1 \mathrm{~s}$ forward and backward as boundary, shift the down-sampled square wave train by frame and calculate its inner product with each spike train $T_{S}^{i}$ of the electrode array SEMG, save the maximum value of the inner product, i.e. the number of common spikes $N_{\mathrm{COM}}^{i}$.

(4) Calculate the $\mathrm{MR}$ of $T_{I}$ and $T_{S}^{i}$ as $\mathrm{MR}^{i}=$ $\frac{2 \cdot N_{\mathrm{COM}}^{i}}{N_{I}+N_{S}^{i}} \cdot 100 \%$, where $N_{I}$ and $N_{S}^{i}$ are the total number of spikes of the concentric needle EMG spike train and the $i$ th SEMG spike train, respectively.

(5) Find the maximum MR, MR $=\max _{i}\left\{\mathrm{MR}^{i}\right\}$; if $\mathrm{MR}>80 \%$, a common spike train is considered to be identified.

Note that if we consider a needle EMG spike train as the reference (or "standard" spike train), the MR is indeed a F1-score measure $\stackrel{49}{9}$ To further understand how the mismatches occurred in a matching pair, we also calculated the false negative rate $(\mathrm{FNR})^{49}$ and the false discovery rate (FDR), $, 50,51$ as defined below, which can be used to measure the proportion of mismatches in the needle EMG spike train and the SEMG spike train, respectively.

$$
\begin{aligned}
& \mathrm{FNR}=\frac{N_{I}-N_{\mathrm{COM}}}{N_{I}} \cdot 100 \%, \\
& \mathrm{FDR}=\frac{N_{S}-N_{\mathrm{COM}}}{N_{S}} \cdot 100 \%,
\end{aligned}
$$

where $N_{I}, N_{S}$ and $N_{\mathrm{COM}}$ have the same definition as used in (1) for MR calculations.

\section{Results}

Figure 2 shows a representative example of automatic decomposition procedures of high-density SEMG signals using the APFP. The decomposition process is comprised of three peel-off steps and each of them is shown in the figure. For clarity, a $1 \mathrm{~s}$ segment of one channel of the electrode array EMG was used for demonstration. For each step, the original signal, the estimated MUAP waveforms and discharge timings of all the identified MUs (after this peel-off step), the reconstructed signal and the residual signal are shown from top to bottom, respectively. For an easy comparison the original SEMG signal is shown in each of the peel-off steps. It can be observed that the original signal has obvious MUAP superposition and it is also contaminated with powerline interference. After each peel-off step, we can observe that the reconstructed signal became progressively closer to the original signal and the residual signal (after subtracting the reconstructed signal from the original signal) became more and more sparse. After three peel-off procedures, 11 MUs were identified, and there was almost only powerline interference left in the residual signal. Note that in every peel-off step, we searched new MUs in the residual signal and used the firing information of all the extracted MUs to estimate their MUAP waveform from the original signal. So, the MUAP template waveforms for each MU were updated after each peeloff step. With increased number of the extracted MUs, the MUAP waveform estimation would be more accurate. As we can observe from the figure, the extracted MUAP templates of the same MUs remained quite consistent during the three peel-off steps, implying the reliability of the MUAP waveform estimation.

Figure 3 shows a representative example of the IEMG decomposition process. The top panel shows the decomposition process. The largest MU (MU 1) can be easily extracted by an appropriate threshold setting, and we obtained the residue signal after subtracting it from the original signal. The same procedure can be applied to extract the second MU (MU 2 ) from the residual signal. However, we cannot easily extract any new MUs from the second residue (after subtracting the two MUs). After applying the valley-seeking clustering we clustered the spikes in the second residue into three clusters. However, by observing the clustering feature space (bottom-left panel), we found that the first cluster (marked by red "+") is more dispersed (i.e. has no clear clustering center) than the other two clusters. Therefore, it was considered to be a noise cluster, while the other two clusters with clear clustering centers were the newly identified MUs. In fact, the spikes from the noise cluster are the residual error of the first two identified MUs after the peel-off steps, which can be 

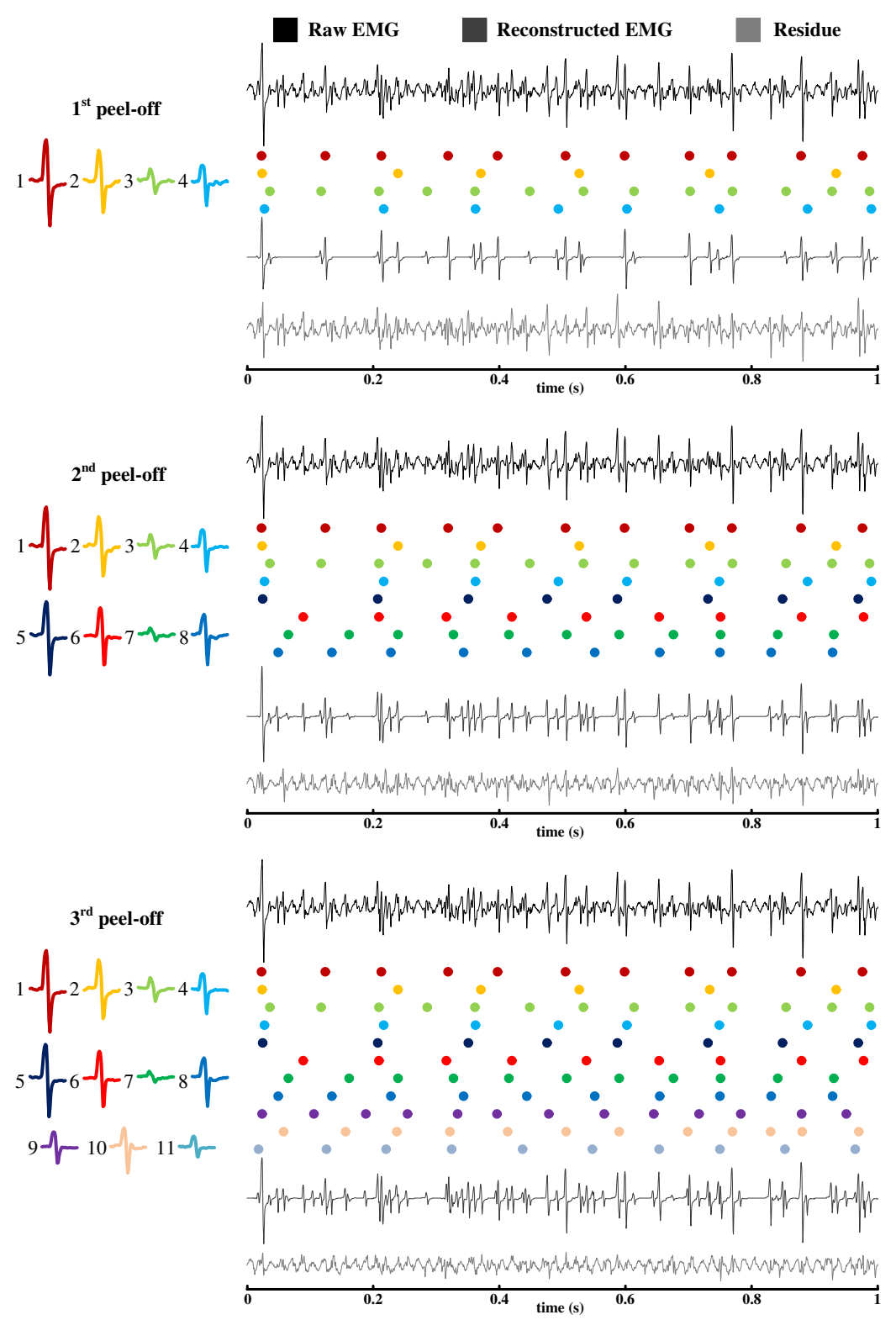

Fig. 2. An example with step-by-step demonstration of the APFP framework. For each of the three peel-off steps, the original signal (of one channel), the estimated MUAP waveform templates, the discharge timings of identified MUs, the reconstructed signal and the residual signal are shown, respectively, from top to bottom.

clearly seen in the zoom-in view of the selected segment (bottom-right panel).

In total, we collected and decomposed 153 trials of simultaneously recorded high-density SEMG and concentric needle EMG signals, among which common MUs were found from 114 trials. For the other 39 trials, either no common MUs were found or the proposed IEMG decomposition method failed to identify the reliable MUs from the concentric needle EMG signals due to heavy MUAP superposition.
Therefore, our data analysis was focused on the 114 trials with common MUs.

Among these 114 trials data, the length of segments used for decomposition analysis varied from $10 \mathrm{~s}$ to $70 \mathrm{~s}(34.1 \pm 8.9 \mathrm{~s})$. Overall, a total of 861 (on average $7.6 \pm 2.7$ per trial) MUs were automatically identified from the high density SEMG signals using the APFP. A total of 217 (on average $1.9 \pm 1.0$ per trial) MUs were identified from the concentric needle EMG. 


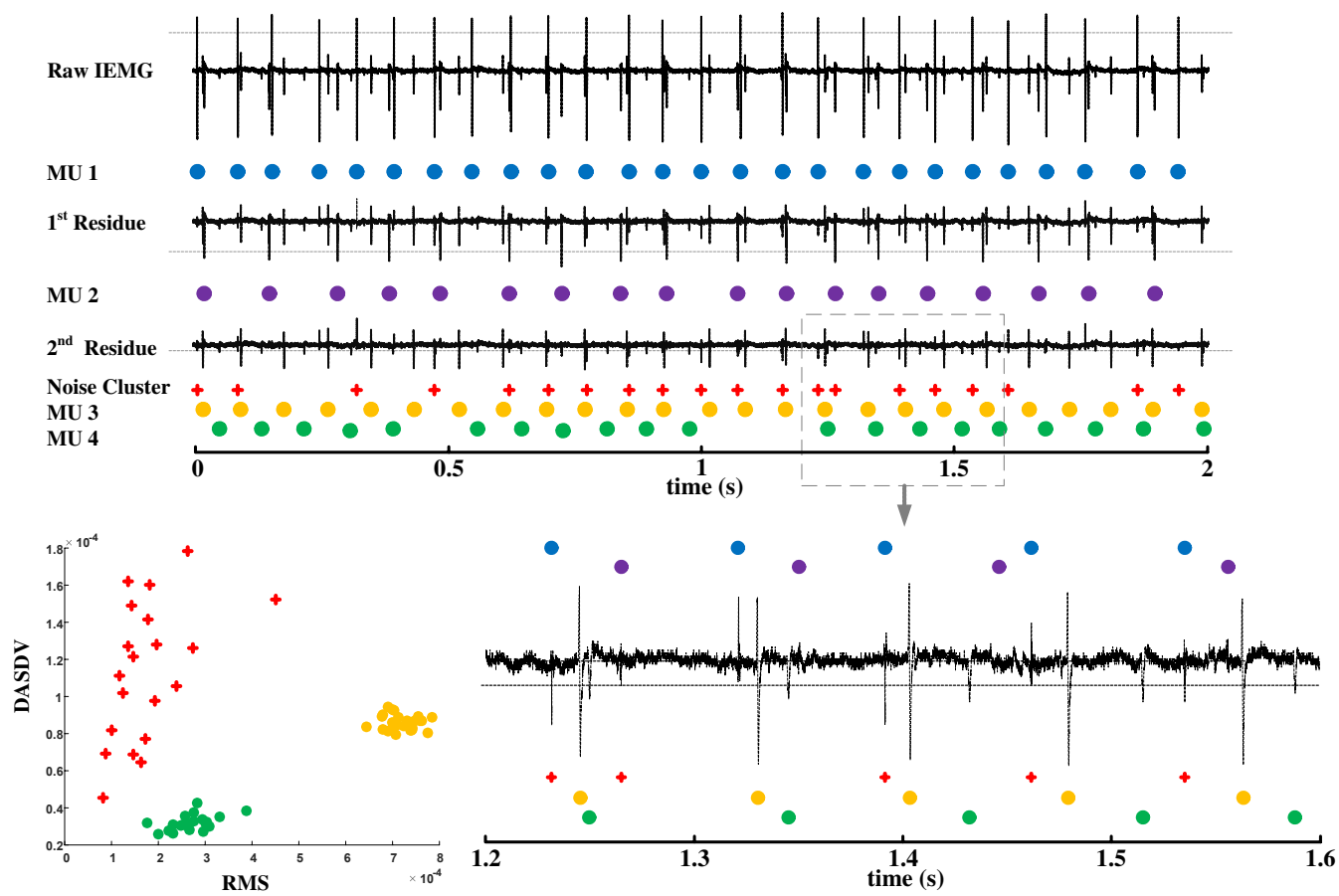

Fig. 3. An example of the decomposition procedure for the IEMG. Top panel: The whole decomposition procedure. Bottom-left panel: The valley-seeking clustering of the extracted spikes from the second residue on feature space; the RMS and the DASDV were used as clustering features. Bottom-right panel: A zoom in view of a selected segment of the second residue $(1.2 \mathrm{~s}$ to $1.6 \mathrm{~s})$.

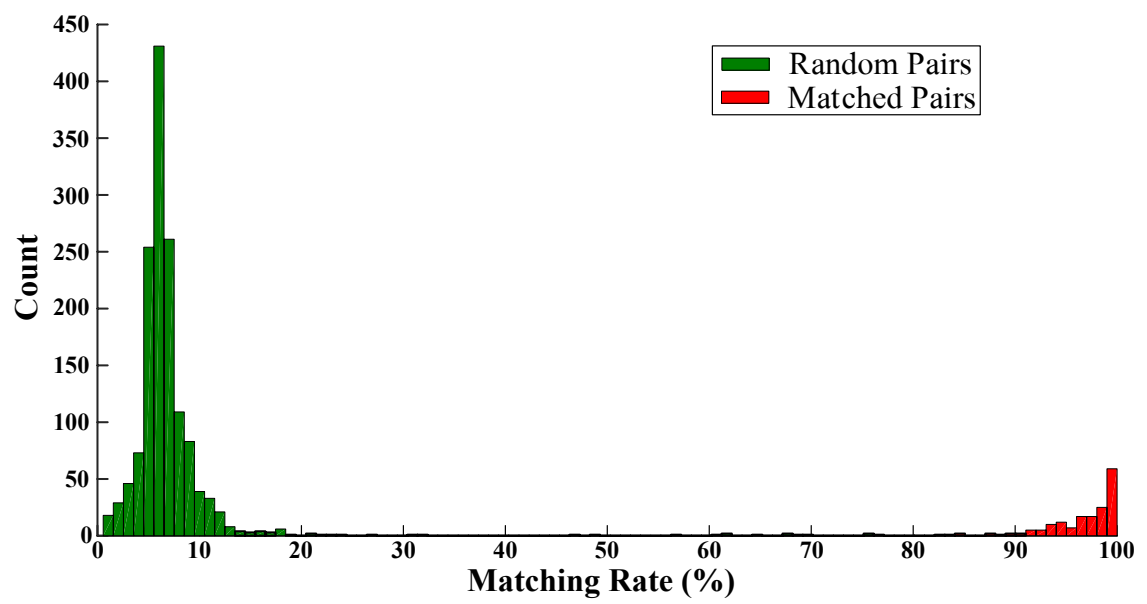

Fig. 4. (Color online) Distribution of MR between random pairs of surface and intramuscular MUAP trains in individual trials. The matched pairs are shown in red bars.

Figure 4 demonstrates the reliability of the inner product matching and the distribution of the MRs, where the MRs of all the random pairs within one trial are calculated and a frequency histogram of MRs across all the 114 trials is shown in the figure. Note that the distribution has a bimodal pattern, where almost all the MRs are distributed within
[0, 20\%] and [80\%, 100\%] (which also supports an appropriate setting of $80 \%$ as MR threshold for finding the common MUs). Leftward, most of the random MRs are under $20 \%$, which indicates that they cannot be common MUs (for example, for $10 \% \mathrm{MR}$, the number of unmatched spikes is 18 times the number of matched spikes according to the calculation of the 


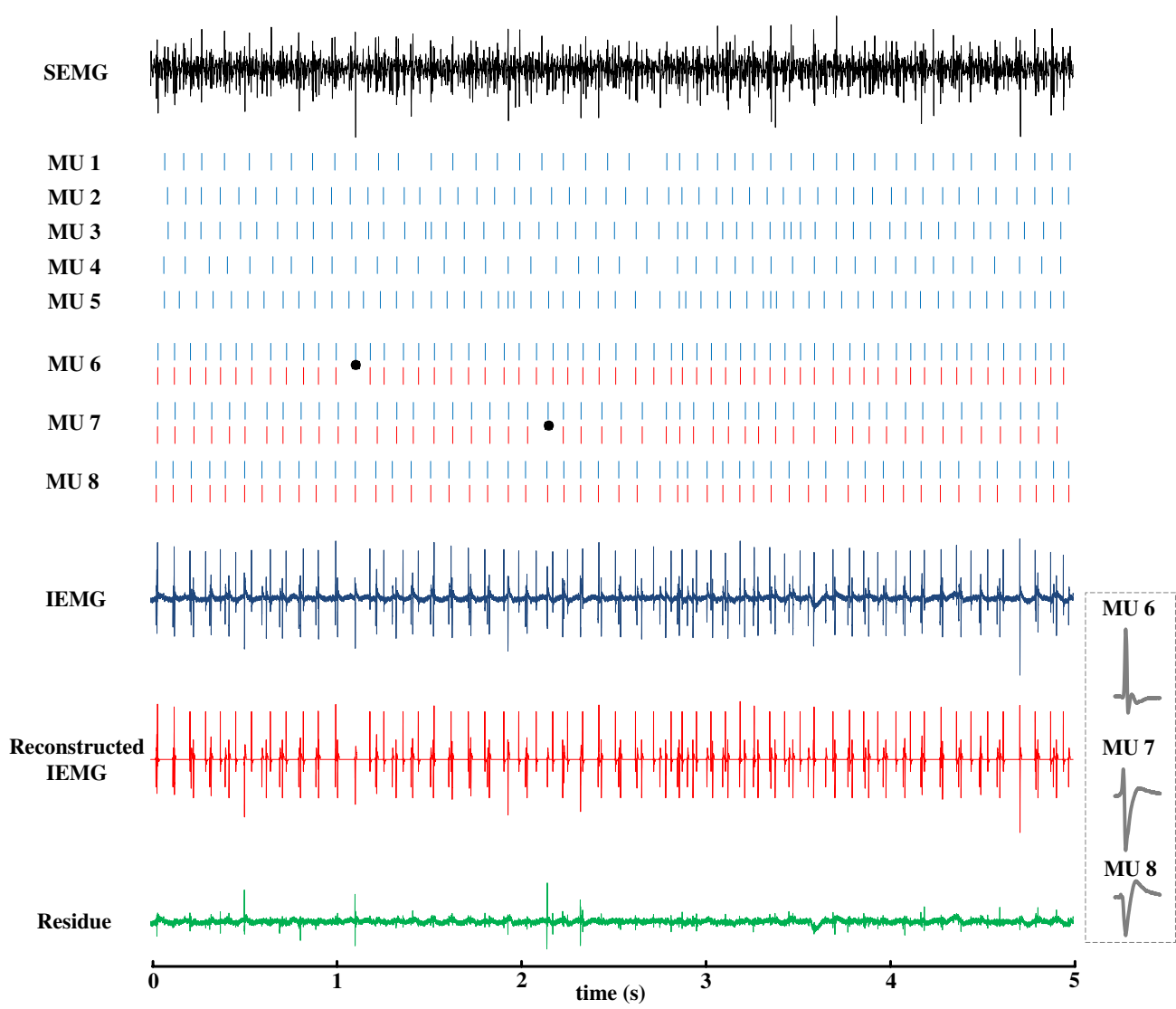

Fig. 5. (Color online) An example of discharge timing comparison for MUs identified from SEMG and IEMG. The blue spike trains represent the results obtained by the APFP framework, and the red ones represent the results obtained from IEMG. Black dots represent the unmatched locations. For clarity, the waveform of identified MUAP, the reconstructed IEMG and the residue are shown in the bottom panel.

MR). Rightward, a total of 168 MUs were accepted as common ones. An average MR for the common MUs was $(96.81 \pm 3.65) \%$; the average FNR and the average FDR were $(2.42 \pm 3.83) \%$ and $(3.80 \pm 5.02) \%$, respectively. It is noted that the MRs of more than half ( 85 pairs) of the 168 common MUs are greater than $98 \%$, indicating that the firing instants of these two spike trains are almost or exactly the same. We also checked those very few pairs with MRs from $20 \%$ to $80 \%$, and found that these situations often occurred in those sparse pairs, which had a relatively small number of firing instants.

An example of a trial with common MUs is shown in Fig. [5 where high-density SEMG and concentric needle EMG signals were independently decomposed. The top panel shows $5 \mathrm{~s}$ SEMG signal from one channel of the electrode array and the automatic decomposition yield using the APFP (blue spikes). The bottom panel shows the simultaneously recorded concentric needle EMG signal, the estimated MUAP waveforms of the three identified MUs, the reconstructed concentric needle EMG signal and the residue. In this example, all three MUs identified from the needle EMG are also found in the SEMG. The comparison of the firing information of the common MUs can be found in the middle panel of the figure, where the blue spike trains represent the automatic decomposition results from the electrode array SEMG signals using the APFP, and the red ones represent the results independently obtained from the concentric needle EMG signal. The corresponding firing spike trains are put together and the locations of inconsistent firing instants are indicated by black dots.

\section{Discussion}

This study presents the two-source validation of the APFP framework for automatic decomposition of 
high density SEMG signals. By utilizing a series of simultaneous surface electrode array and IEMG recordings from the human FDI muscle, the agreement of discharge timings was analyzed for the common MUs independently decomposed from each recording type. Comparison of the decomposition of electrode array SEMG and IEMG signals yields a total of 168 common MUs with an average MR of $96.81 \%$. These outcomes confirm that the APFP can reliably decompose at least a subset of MUs in high density SEMG signals recorded from the FDI muscle during low contraction levels.

High density SEMG has been emerging as a promising tool in clinical neurophysiology, 42 which can provide valuable information (e.g. muscle fiber conduction velocity, MU territory, innervation zone localization) to supplement needle EMG examination. The advance in high density surface EMG decomposition will further promote its application in both research and clinical laboratories.

A primary feature of the current study is that we applied a strategy or protocol commonly used in clinical EMG examination (e.g. by providing a resistance force to the examined muscle and instructing the subject to perform a series of isometric contractions; by applying concentric needle electrode to record IEMG). By contrast, in the previous two-source validation studies, 4244 standard laboratory load cell setup was required and subjects were often asked to perform precise muscle force control or follow a strict trapezoidal force protocol at different contraction levels. Associated with this protocol, fine-wire electrode was always used for IEMG recording. In addition, compared with previous studies, $\underline{42,44}$ the duration of the EMG signals recorded in the current study is relatively long (from $10 \mathrm{~s}$ to more than $1 \mathrm{~min})$. Note that the longer the analyzed signal, the harder two MUs can be matched, thus avoiding a false high random MR caused by the short signal duration.

In this two-source validation study, the contraction force was constrained to low levels to ensure accurate decomposition of the concentric needle EMG signals. We aimed to extract the most reliable MUs (i.e. with no or little superposition and distinctive amplitudes) during concentric needle EMG decomposition, relying on MUAP waveform information (while the MU firing information was not used). Those MUs which are difficult to separate due to
MUAP superposition were discarded to avoid inaccurate spike identifications. Under this strategy, we found an average of about two common MUs per trial, allowing to estimate the accuracy of the APFP for automatically decomposing these MUs.

To describe the nature of the mismatched spikes of the common MUs, the FNR and FDR were calculated. The results indicate that the mismatches are not always from one side. The average FDR is slightly higher than FNR, suggesting that relatively more mismatches are associated with the APFP. However, it should be noted that the inconsistence in discharge instants of the common MUs does not necessarily mean the SEMG decomposition is wrong. An example demonstrating this can be found in Fig. 5. For the two inconstant discharge spikes shown in Fig. 5 (where the two black dots are located), it is more likely that the two spikes were not able to be identified from the single channel concentric needle EMG due to MUAP superposition.

The limitations of the two-source validation studies (including the current study) should be acknowledged. To allow reliable decomposition of IEMG, the experimental protocols for two-source validation studies are usually limited at low muscle contraction levels. Therefore, SEMG decomposition performance can only be assessed at low muscle contraction levels. In addition, two-source validation provides a direct evaluation only for those common MUs extracted from the IEMG and SEMG signals. However, the common MUs typically account for a small portion of MUs extracted from the SEMG. The difficulty in obtaining a larger number of common MUs can be due to both signal recording and processing limitations. It requires that surface and intramuscular electrodes record an overlapping muscle volume with simultaneously active MUs, which can be extracted by both IEMG and SEMG decomposition algorithms.

In fact, there is hardly a solution for direct evaluation of all the decomposed MUs from the experimental SEMG signals (particularly those with heavy MUAP superpositions). Given that the APFP is an automatic iterative method applying a peel-off strategy, the effectiveness of the peel-off is based on a correct estimation of the MU discharge timings and MUAP waveforms. Otherwise, cumulative errors will be involved, jeopardizing the decomposition performance. Assuming that the estimated 
firing instants and MUAP waveforms of the subtracted MUs are correct, then the residual signal tends to become more and more sparse (and ultimately close to background baseline), where the superposed MUAPs can be gradually detangled and start to emerge. Although this is the pattern we can sometimes observe from a step-by-step demonstration of the peel-off procedures, a more systematic and quantitative assessment of the accuracy for all of the decomposed MUs is still lacking for experimental signals. For example, Fig. 2 shows how a total of 11 MUs were progressively decomposed from the SEMG using a peel-off strategy, where the final residue almost included only the powerline interference. The accuracy of two MUs in this example can be directly validated by the two-source validation analysis. Although the high MRs of the two common MUs provide a confidence in the general validity of the SEMG decomposition, it still remains an issue to be solved how to provide a direct and quantitative evaluation for the remaining nine MUs.

Given a relatively small number of subjects recruited in this study, we performed recordings bilaterally for some subjects in order to have sufficient data for the validation purpose. Although a stroke subject participated in this study, we did not observe any noticeable difference between this stroke subject and the neurologically intact subjects, in terms of the two-source validation outcomes. The present two-source validation was only performed for the FDI muscle. Further evaluation of the SEMG decomposition performance is needed for different types and conditions of muscles (such as proximal large muscles, diseased muscles).

\section{Acknowledgments}

This work was supported by the National Natural Science Foundation of China Under Grant No. 61771444, the Guangzhou Science and Technology Programme Under Grant No. 201704030039, and the National Institutes of Health of the U.S. Department of Health and Human Services under Grant No. R21NS093727.

\section{Appendix A. The Implementation of the APFP Framework ${ }^{24}$}

Step 1 Initialize the residue as the raw SEMG data, and the spike trains set $\psi$ as null set;
Step 2 Run the parallel FastICA on the extended residue, extract and sort the spike trains;

Step 3 For each spike train obtained in Step 2, repeatedly run constrained FastICA until the extracted spike train from output is not changed;

Step 4 Automatically judge the reliability of all the filtered spike trains from Step 3 and store the reliable spike trains to the set $\psi$;

Step 5 For each channel of the raw SEMG data, use all the spike trains of $\psi$ to estimate the MUAP waveforms, then subtract these identified MUAP trains from the raw SEMG data (i.e. a peel-off procedure);

Step 6 Update the residue, then go to Step 2;

Step 7 Output $\psi$ if no reliable spike trains emerge or if the program reaches the preset termination conditions.

\section{Appendix B. The Process of Valley-Seeking Clustering 47}

Definition: Suppose $x$ and $x^{\prime}$ to be the two points in the data set. If $x^{\prime}$ is the $k$ th neighbor of $x$ (according to the distance), we say that $x^{\prime}$ has the neighbor number $(\mathrm{NN})$ of $k$ with respect to $x$, which is denoted as $\mathrm{NN}\left(x, x^{\prime}\right)=k$. Similarly, let $\mathrm{NN}\left(x^{\prime}, x\right)=$ $l$. Then we denote the $i$ th neighbor of $x$ as $x_{(i)}$, i.e. $\mathrm{NN}\left(x, x_{(i)}\right)=i$, and $\mathrm{NN}\left(x_{(i)}, x\right)=a_{(i)}, i=$ $1,2, \ldots, k-1$; Similarly, denote the $j$ th neighbor of $x^{\prime}$ as $x_{(j)}^{\prime}$, i.e. $\mathrm{NN}\left(x^{\prime}, x^{\prime}{ }_{(j)}\right)=j$ and $\mathrm{NN}\left(x^{\prime}{ }_{(j)}, x^{\prime}\right)=b_{(j)}$, $j=1,2, \ldots, l-1$.

Steps:

(1) Input parameters $t_{1}, t_{2}$ and $t_{3}$, calculate the Euclidean distance matrix $D=\left(d_{i j}\right)$, dimensionality $m$.

(2) Determine the NN matrix $L=\left(l_{i j}\right)$, where $l_{i j}=$ $\mathrm{NN}\left(x_{i}, x_{j}\right)$.

(3) Determine matrix $S=\left(s_{i j}\right)$, where $s_{i j}=\frac{l_{i j}+l_{j i}}{2}$.

(4) Estimate NND (normalized density derivative) matrix $J=\left(J_{i j}\right)$, where $J_{i j}=\frac{\left|l_{i j}-l_{j i}\right|}{s_{i j}^{1+1 m}}, s_{i j} \leq t_{1}$.

(5) Estimate the convexity $D 2=\left(d 2_{i j}\right)$, where

$$
d 2_{i j}=\frac{l_{i j} \sum_{i=1}^{k-1} a_{(i)}+l_{j i} \sum_{j=1}^{l-1} b_{(j)}}{l_{i j} \sum_{i=1}^{k-1} i+l_{j i} \sum_{j=1}^{l-1} j}, s_{i j} \leq t_{1} .
$$


(6) Determine the discretized connectivity matrix $C=\left(c_{i j}\right)$, where $c_{i j}=I\left(s_{i j} \leq t_{1}, J_{i j} \leq\right.$ $\left.t_{2}, d 2_{i j} \leq t_{3}\right)$.

(7) Assign cluster labels to observations according to the discretized connectivity matrix $C$.

In this study, we set parameters $t_{1}, t_{2}$ and $t_{3}$ as 20 , 0.3 and 2, respectively. The RMS and the DASDV were used as clustering features.

\section{References}

1. G. Phongsamart and J. J. Wertsch, Quantitative electromyography, Phys. Med. Rehabil. Clin. 14(2) (2003) 231-241.

2. C. Farkas, A. Hamilton-Wright, H. Parsaei and D. W. Stashuk, A review of clinical quantitative electromyography, Critical Reviews? in Biomed. Eng. 38(5) (2010) 467-485.

3. M. de Carvalho, A. Turkman and M. Swash, Motor unit firing in amyotrophic lateral sclerosis and other upper and lower motor neurone disorders, Clin. Neurophysiol. 123(11) (2012) 2312-2318.

4. C. J. De Luca and P. Contessa, Hierarchical control of motor units in voluntary contractions, J. Neurophysiol. 107(1) (2011) 178-195.

5. K. D. Bergmeister, I. Vujaklija, S. Muceli, A. Sturma, L. A. Hruby, C. Prahm, O. Riedl, S. Salminger, K. Manzano-Szalai, M. Aman et al., Broadband prosthetic interfaces: Combining nerve transfers and implantable multichannel emg technology to decode spinal motor neuron activity, Front. Neurosci. 11 (2017) 421.

6. A. Ortiz-Rosario and H. Adeli, Brain-computer interface technologies: From signal to action, Rev. Neurosci. 24(5) (2013) 537-552.

7. A. Burns, H. Adeli and J. A. Buford, Braincomputer interface after nervous system injury, $\mathrm{Neu}$ roscientist 20(6) (2014) 639-651.

8. R. Liu, Y. Wang, G. I. Newman, N. V. Thakor and S. Ying, Eeg classification with a sequential decisionmaking method in motor imagery bci, Int. J. Neural Syst. 27(8) (2017) 1750046.

9. F. Xu, W. Zhou, Y. Zhen, Q. Yuan and Q. Wu, Using fractal and local binary pattern features for classification of ecog motor imagery tasks obtained from the right brain hemisphere, Int. J. Neural Syst. 26(6) (2016) 1650022.

10. D. Stashuk, Emg signal decomposition: How can it be accomplished and used? J. Electromyogr. Kinesiol. 11(3) (2001) 151-173.

11. K. C. McGill, Z. C. Lateva and H. R. Marateb, Emglab: An interactive emg decomposition program, J. Neurosci. Methods 149(2) (2005) 121133.
12. H. Parsaei, D. W. Stashuk, S. Rasheed, C. Farkas and A. Hamilton-Wright, Intramuscular emg signal decomposition, Critical Reviews? Biomed. Eng. 38(5) (2010) 435-465.

13. S. H. Nawab, R. P. Wotiz and C. J. De Luca, Decomposition of indwelling emg signals, J. Applied Physiol. 105(2) (2008) 700-710.

14. P. Zhou and W. Z. Rymer, Muap number estimates in surface emg: Template-matching methods and their performance boundaries, Ann. Biomed. Eng. 32(7) (2004) 1007-1015.

15. R. Merletti, M. Aventaggiato, A. Botter, A. Holobar, H. Marateb and T. M. Vieira, Advances in surface emg: Recent progress in detection and processing techniques, Critical Reviews? Biomed. Eng. 38(4) (2010) 305-345.

16. B. G. Lapatki, J. P. Van Dijk, I. E. Jonas, M. J. Zwarts and D. F. Stegeman, A thin, flexible multielectrode grid for high-density surface emg, J. Appl. Physiol. 96(1) (2004) 327-336.

17. G. Drost, D. F. Stegeman, B. G. van Engelen and M. J. Zwarts, Clinical applications of high-density surface emg: A systematic review, J. Electromyogr. Kinesiol. 16(6) (2006) 586-602.

18. Y. Liu, Y. Ning, S. Li, P. Zhou, W. Z. Rymer and Y. Zhang, Three-dimensional innervation zone imaging from multi-channel surface emg recordings, Int. J. Neural Syst. 25(6) (2015) 1550024.

19. B. U. Kleine, J. P. van Dijk, B. G. Lapatki, M. J. Zwarts and D. F. Stegeman, Using two-dimensional spatial information in decomposition of surface emg signals, J. Electromyogr. Kinesiol. 17(5) (2007) 535548.

20. A. Holobar and D. Zazula, Multichannel blind source separation using convolution kernel compensation, IEEE Trans. Signal Proces. 55(9) (2007) 4487-4496.

21. A. Holobar and D. Farina, Blind source identification from the multichannel surface electromyogram, Physiol. Meas. 35(7) (2014) R143.

22. A. Holobar, D. Farina, M. Gazzoni, R. Merletti and D. Zazula, Estimating motor unit discharge patterns from high-density surface electromyogram, Clin. Neurophysiol. 120(3) (2009) 551-562.

23. M. Chen and P. Zhou, A novel framework based on fastica for high density surface emg decomposition, IEEE Trans. Neural Syst. Rehabil. Eng. 24(1) (2016) $117-127$.

24. M. Chen, X. Zhang, X. Chen and P. Zhou, Automatic implementation of progressive fastica peel-off for high density surface emg decomposition, IEEE Trans. Neural Syst. Rehabil. Eng. 26(1) (2017) 144152.

25. A. Hyvärinen, J. Karhunen and E. Oja, Independent Component Analysis (John Wiley \& Sons, US, 2004).

26. R. Fu and H. Wang, Detection of driving fatigue by using noncontact emg and ecg signals measurement system, Int. J. Neural Syst. 24(3) (2014) 1450006. 
27. I. Rembado et al., Independent component decomposition of human somatosensory evoked potentials recorded by micro-electrocorticography, Int. J. Neural Syst. 27(4) (2017) 1650052.

28. L. Khedher, I. A. Illán, J. M. Górriz, J. Ramírez, A. Brahim and A. Meyer-Baese, Independent component analysis-support vector machine-based computer-aided diagnosis system for alzheimer's with visual support, Int. J. Neural Syst. 27(3) (2017) 1650050.

29. G. R. Naik, A. H. Al-Timemy and H. T. Nguyen, Transradial amputee gesture classification using an optimal number of semg sensors: An approach using ica clustering, IEEE Trans. Neural Syst. Rehabil. Eng. 24(8) (2016) 837-846.

30. G. R. Naik, S. Arjunan and D. Kumar, Applications of ica and fractal dimension in semg signal processing for subtle movement analysis: A review, Australas. Phys. Eng. Sci. Med. 34(2) (2011) 179-193.

31. A. Hyvarinen, Fast and robust fixed-point algorithms for independent component analysis, IEEE Trans. Neural Netw. 10(3) (1999) 626-634.

32. H. Nakamura, M. Yoshida, M. Kotani, K. Akazawa and T. Moritani, The application of independent component analysis to the multi-channel surface electromyographic signals for separation of motor unit action potential trains: Part I - Measuring techniques, J. Electromyogr. Kinesiol. 14(4) (2004) 423-432.

33. H. Nakamura, M. Yoshida, M. Kotani, K. Akazawa and T. Moritani, The application of independent component analysis to the multi-channel surface electromyographic signals for separation of motor unit action potential trains: Part II - Modelling interpretation, J. Electromyogr. Kinesiol. 14(4) (2004) 433-441.

34. X. Ren, X. Hu, Z. Wang and Z. Yan, Muap extraction and classification based on wavelet transform and ica for emg decomposition, Med. Biol. Eng. Comput. 44(5) (2006) 371.

35. G. A. Garcia, R. Okuno and K. Azakawa, A decomposition algorithm for surface electrode-array electromyogram, IEEE Eng. Med. Biol. Mag. 24(4) (2005) 63-72.

36. M. Chen, A. Holobar, X. Zhang and P. Zhou, Progressive fastica peel-off and convolution kernel compensation demonstrate high agreement for high density surface emg decomposition, Neural Plast. 2016 (2016) 3489540.

37. S. D. Nandedkar, Models and simulations in electromyography, Muscle Nerve 25(S11) (2002) S46-S54.

38. K. McGill, Surface electromyogram signal modelling, Med. Biol. Eng. Comput. 42(4) (2004) 446454.
39. M. M. Lowery, R. F. Weir and T. A. Kuiken, Simulation of intramuscular emg signals detected using implantable myoelectric sensors (imes), IEEE Trans. Biomed. Eng. 53(10) (2006) 1926-1933.

40. V. Carriou, S. Boudaoud, J. Laforet and F. S. Ayachi, Fast generation model of high density surface emg signals in a cylindrical conductor volume, Comput. Biol. Med. 74 (2016) 54-68.

41. B. Mambrito and C. J. De Luca, A technique for the detection, decomposition and analysis of the emg signal, Electroencephalogr. Clin. Neurophysiol. 58(2) (1984) 175-188.

42. A. Holobar, M. A. Minetto, A. Botter, F. Negro and D. Farina, Experimental analysis of accuracy in the identification of motor unit spike trains from highdensity surface emg, IEEE Trans. Neural Syst. Rehabil. Eng. 18(3) (2010) 221-229.

43. H. R. Marateb, K. C. McGill, A. Holobar, Z. C. Lateva, M. Mansourian and R. Merletti, Accuracy assessment of ckc high-density surface emg decomposition in biceps femoris muscle, J. Neural Eng. 8(6) (2011) 066002.

44. X. Hu, W. Z. Rymer and N. L. Suresh, Accuracy assessment of a surface electromyogram decomposition system in human first dorsal interosseus muscle, J. Neural Eng. 11(2) (2014) 026007.

45. X. Li, A. Holobar, M. Gazzoni, R. Merletti, W. Z. Rymer and P. Zhou, Examination of poststroke alteration in motor unit firing behavior using highdensity surface emg decomposition, IEEE Trans. Biomed. Eng. 62(5) (2015) 1242-1252.

46. X. Li, A. Suresh, P. Zhou and W. Z. Rymer, Alterations in the peak amplitude distribution of the surface electromyogram poststroke, IEEE Trans. Biomed. Eng. 60(3) (2013) 845-852.

47. C. Zhang, X. Zhang, M. Q. Zhang and Y. Li, Neighbor number, valley seeking and clustering, Pattern Recognit. Lett. 28(2) (2007) 173-180.

48. S. Karimimehr, H. R. Marateb, S. Muceli, M. Mansourian, M. A. Mañanas and D. Farina, A real-time method for decoding the neural drive to muscles using single-channel intra-muscular emg recordings, Int. J. Neural Syst. 27(6) (2017) 1750025.

49. D. M. Powers, Evaluation: From Precision, Recall and F-Measure to Roc, Informedness, Markedness and Correlation (Bioinfo Publications, Pune, India, 2011)

50. Y. Benjamini and Y. Hochberg, Controlling the false discovery rate: A practical and powerful approach to multiple testing, J. Royal Stat. Soc. B Methodol. 57(1) (1995) 289-300.

51. J. Xi and A. Li, Discovering recurrent copy number aberrations in complex patterns via non-negative sparse singular value decomposition, IEEE/ACM Trans. Comput. Biol. Bioinf. 13(4) (2016) 656-668. 